\title{
Safety and efficacy of sorafenib in the treatment of hepatocellular carcinoma
}

This article was published in the following Dove Press journal:

OncoTargets and Therapy

10 November 2009

Number of times this article has been viewed

\author{
Khaldoun Almhanna \\ Philip A Philip \\ Department of Hematology \\ and Oncology, Karmanos Cancer \\ Institute, Wayne State University, \\ Detroit, MI 4820I, USA
}

Correspondence: Philip A Philip 4-HWCRC, 4I00 John R Street, Detroit, Michigan 4820I, USA

$\mathrm{Tel}+$ I 3/3-576-8746

Fax + I 3/3-576-8729

Email philipp@karmanos.org

\begin{abstract}
Hepatocellular carcinoma (HCC) is frequently diagnosed in the setting of chronic liver disease and cirrhosis. The median survival after diagnosis is dismal. The treatment options that may offer cure are either resection or liver transplantation. Unfortunately most patients are not eligible for either treatment modality at diagnosis because of advanced stage and underlying liver dysfunction. Until recently, there was no effective systemic therapy for patients with advanced HCC. Sorafenib, an oral multikinase inhibitor of the vascular endothelial growth factor receptor, the platelet-derived growth factor receptor and Raf, has shown antitumor activity in patients with advanced HCC in phase III trials. Although objective response is not common, sorafenib promotes disease stabilization and improves overall survival. Sorafenib is well tolerated with a favorable toxicity profile. In this article we review the efficacy and safety data for sorafenib in patients with advanced HCC.
\end{abstract}

Keywords: sorafenib, hepatocellular carcinoma, hand-foot syndrome

\section{Introduction}

Hepatocellular carcinoma (HCC) comprises the third highest cause of cancer death worldwide. ${ }^{1}$ Surgical resection offers a cure; however most patients are ineligible for resection at the time of diagnosis because of tumor extent and underlying liver disease. Prognosis remains dismal in unresectable patients with a median survival of 3 to 6 months after diagnosis. ${ }^{2}$ Liver transplantation in properly selected patients with unresectable tumors offers the potential for cure and the treatment of the underlying liver disease.

Conventional therapeutic options for advanced HCC are limited. HCC is resistant to cytotoxic chemotherapy due in part to the high rate of expression of drug resistance genes. A small percentage of patients with $\mathrm{HCC}$ benefit from cytotoxic therapy, and until recently no improved survival in this patient population had been demonstrated.

Sorafenib is an oral small molecule multikinase inhibitor with antiproliferative and antiangiogenic effects (Figure 1). It acts predominantly by inhibiting the activity of the serine/threonine kinases c-Raf and B-Raf and vascular endothelial growth factor receptors (VEGFR) 1, 2 and 3. Other targets include the mitogenactivated protein kinases MEK and ERK; the platelet derived growth factor receptor (PDGFR); the cytokine receptor c-KIT; the receptor tyrosine kinases Flt-3 and RET; and the Janus kinase/signal transducer and activator of transcription (JAK/STAT) pathway. ${ }^{3}$ The Raf/MEK/ERK intracellular signaling pathway and the VEGFR and 


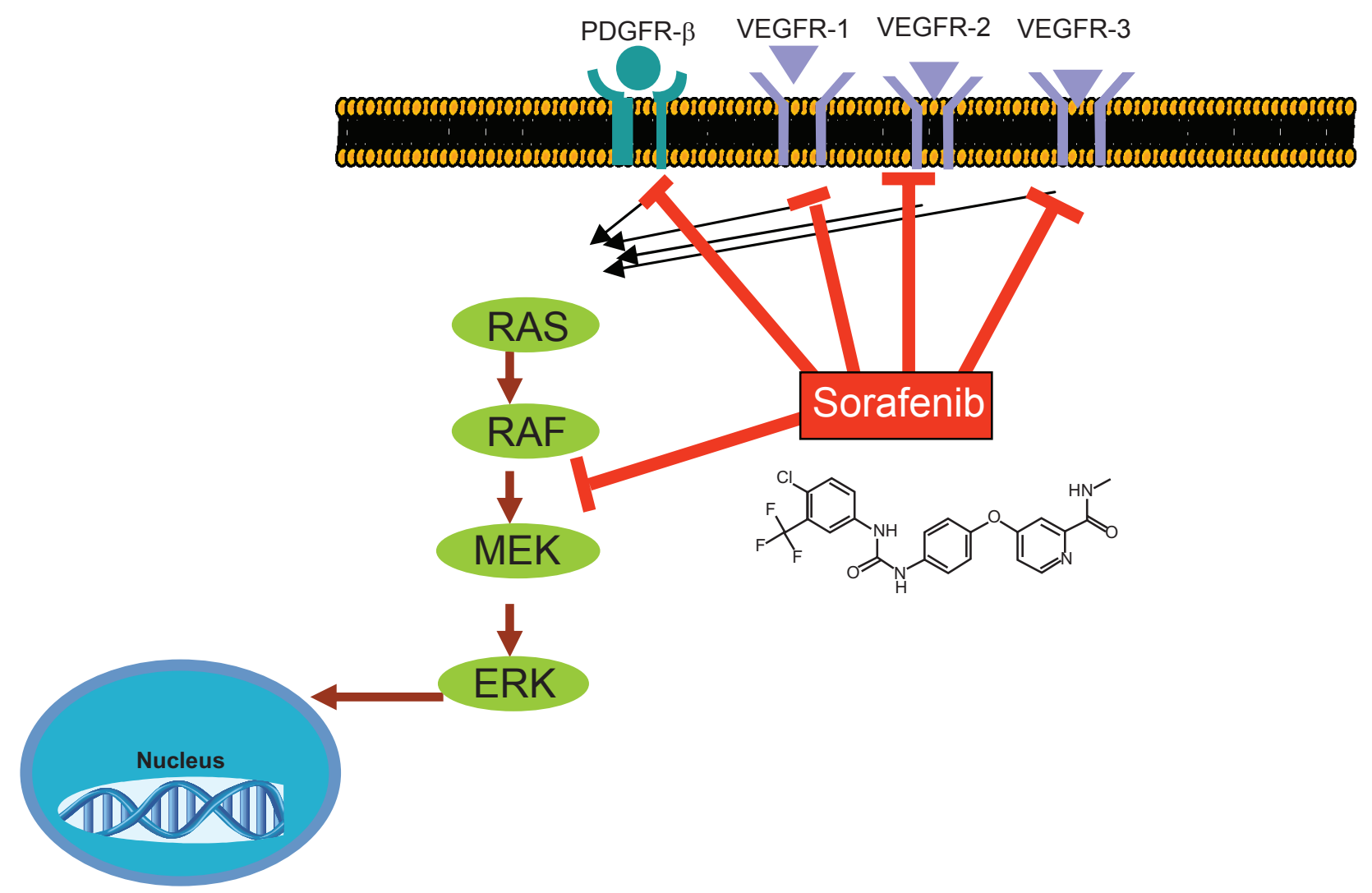

Figure I Sorafenib acts by inhibiting the activity of c-Raf and B-Raf; vascular endothelial growth factor receptors (VEGFR); the mitogen-activated protein kinases MEK and ERK; and platelet derived growth factor receptors (PDGFR).

PDGFR extracellular receptors have been implicated in the pathogenesis of hepatocellular carcinoma, prompting the investigation of sorafenib in advanced HCC. In addition the hypervascularity of HCC suggests that strategies targeting angiogenesis may be worthwhile in controlling disease progression. ${ }^{4-7}$

\section{Efficacy of sorafenib in patients with advanced hepatocellular carcinoma}

\section{Phase I and II studies}

The efficacy of sorafenib in HCC was initially suggested by a phase I trial of sorafenib in advanced refractory solid tumors $^{8}$ in which one patient with HCC showed a partial response at $400 \mathrm{mg}$ twice daily continuous sorafenib lasting more than 6 months. Three out of 6 patients with HCC in that study showed disease stabilization lasting for months. Another phase I trial ${ }^{9}$ evaluated the pharmacokinetics, safety, and efficacy of sorafenib in Japanese patients with HCC. Twenty-seven patients with unresectable HCC, ChildPugh status A (CPA) or B (CPB), were treated. Among the
24 patients evaluable for tumor response, 1 patient (4\%) achieved a partial response, 20 (83\%) had stable disease, and $3(13 \%)$ had progressive disease. The median time to progression was 4.9 months and the median overall survival was 15.6 months. Subsequently, a phase II trial of sorafenib in patients with advanced HCC was conducted. ${ }^{10}$ One hundred and thirty patients with inoperable $\mathrm{HCC}$ and CPA or B were treated with sorafenib $400 \mathrm{mg}$ twice daily. Three (2.2\%) patients achieved partial response (PR), $8(5.8 \%)$ had a minor response, and $46(33.6 \%)$ had stable disease for at least 16 weeks. Median time to progression (TTP) was 4.2 months, and median overall survival (OS) was 9.2 months. Despite a shorter course of therapy for patients with CPB (12.9 weeks) compared to CPA (24.9 weeks), sorafenib discontinuation rates (CPA $31 \%$ vs CPB $21 \%$ ) and dose reduction (CPA 31\% vs CPB 21\%) were similar in both group. Median TTP for patients with CPA was 21 weeks compared to 13 week in CPB patients. Overall survival for patients with CPA and CPB was 41 weeks 14 weeks respectively. ${ }^{11}$ Another phase II open label study of single-agent sorafenib was conducted in hepatitis B-endemic Asian population with HCC. ${ }^{12}$ Fifty-one patients 
were enrolled. The overall median progression-free survival was 3 months and OS was 5 months. Response rate was modest, $8 \%$ of patients achieving PR and $18 \%$ had stable disease for at least 12 weeks. Patients without extrahepatic spread (EHS), particularly lung metastases, were more likely to benefit from the treatment.

\section{Phase III trials (Table I) SHARP study}

On the basis of the above phase II studies, a double-blind, placebo-controlled phase III trial of sorafenib in advanced HCC was conducted (Sorafenib HCC Assessment Randomized Protocol Trial: SHARP trial). ${ }^{13}$ Six hundred and twenty patients with advanced HCC were randomized to receive either sorafenib $400 \mathrm{mg}$ twice daily or placebo. Primary endpoints were OS and time to symptomatic progression (TTSP). Secondary endpoint was time to radiologic progression and safety. The study was stopped after the second planned interim analysis at which 321 deaths had occurred. Median OS was significantly longer in the sorafenib-treated patients (10.7 vs 7.9 months) as was median time to radiologic progression (5.5 vs 2.8 months). Time to symptomatic progression was similar in both

Table I Patient characteristics and outcome in phase III clinical trials of sorafenib versus placebo in advanced HCC

\begin{tabular}{|c|c|c|c|c|}
\hline \multirow[t]{2}{*}{ Variable } & \multicolumn{2}{|l|}{ SHARP } & \multicolumn{2}{|c|}{ Asia-Pacific } \\
\hline & Sorafenib & Placebo & Sorafenib & Placebo \\
\hline No of patients & 299 & 303 & 150 & 76 \\
\hline Age (year) & 64.9 & 66 & 51 & 52 \\
\hline HCV infection (\%) & 29 & 27 & 11 & 4 \\
\hline HBV infection (\%) & 19 & 18 & 70 & 77 \\
\hline \multicolumn{5}{|l|}{ Child-Pugh class } \\
\hline $\mathrm{A}(\%)$ & 95 & 98 & 97.3 & 97.4 \\
\hline B (\%) & 5 & 2 & 2.7 & 2.6 \\
\hline \multicolumn{5}{|l|}{ PS (\%) } \\
\hline 0 & 54 & 54 & 25 & 27 \\
\hline 1 & 38 & 39 & 69 & 67 \\
\hline 2 & 8 & 7 & 5 & 5 \\
\hline Median OS (months) & 10.7 & 7.9 & 6.5 & 4.2 \\
\hline Median TTP (months) & 5.5 & 2.8 & 2.8 & 1.4 \\
\hline Response rate (\%) & 2 & 1 & 3 & 1 \\
\hline Complete response & 0 & 0 & 0 & 0 \\
\hline Partial response & 2 & 1 & 3.3 & 1.3 \\
\hline Stable disease & 7I & 67 & 54 & 27 \\
\hline DCR & 43 & 32 & 35 & 16 \\
\hline
\end{tabular}

Abbreviations: $\mathrm{HCV}$, hepatitis $\mathrm{C}$ virus; $\mathrm{HBV}$, hepatitis B virus; OS, overall survival; TTP, time to progression; DCR, disease control rate; PS, performance status. groups (4.1 vs 4.9 months). Two percent of patients in the sorafenib arm had a partial response compared to $1 \%$ of patients in the placebo group. Based on these results sorafenib was approved in the United States and became the standard of care for patients with advanced HCC.

An exploratory subgroup analysis showed a survival benefit for sorafenib over placebo regardless of HCV status, ECOG performance status (PS), EHS/microscopic vascular invasion, and prior curative treatment.

Of the 602 patients enrolled in the SHARP study, 178 patients were positive for hepatitis $\mathrm{C}$ virus infection (HCV), 93 patients in the sorafenib arm and 85 in the placebo arm. In the HVC positive subgroup the median overall survival was higher in the sorafenib group (14.0 vs 7.9 months) as was median time to radiologic progression by independent review (7.6 vs 2.8 months). The disease control rate (DCR), defined as the percentage of patients who had a best-response rating of complete or partial response or stable disease that was maintained for at least 28 days after the first demonstration of that rating on independent radiologic review, was higher in the sorafenib group also (44 vs 31\%). ${ }^{14}$

The influence of PS on efficacy of sorafenib in the SHARP trial was reported. Out of 602 patients enrolled on the study, 325 had ECOG PS 0 at baseline and 277 had PS 1-2. Among patients with ECOG PS 0, the median OS was higher in the sorafenib group (13.3 vs 8.8 months) as was the median time to progression (5.5 vs 2.9 months). Among patients with ECOG PS 1-2, the median OS (8.9 vs 5.6 months) and the median time to progression (3.5 vs 2.8) were also higher in the sorafenib group, indicating that sorafenib prolonged OS and TTP in patients with advanced HCC irrespective of ECOG PS. ${ }^{15}$

The effect of macroscopic vascular invasion (MVI) and/or EHS, on the efficacy of sorafenib in the SHARP study was also evaluated. In this subanalysis, 421/602 patients had MVI and/or EHS. Patients with MVI and/or EHS treated with sorafenib had a higher OS (8.9 vs 6.7 months) and longer TTP (4.1 vs 2.7 months) than patients treated with placebo; similarly patients without MVI and/or EHS had OS of 14.5 months in sorafenibtreated patients versus 10.2 months in patients treated with placebo. TTP was longer in sorafenib-treated patients as well (9.6 vs 4.3 months). ${ }^{16}$

The effects of previous liver-directed treatment on survival in the SHARP trial population was analyzed; in this subanalysis, 158 patients (81 sorafenib, 77 placebo) underwent prior curative treatment and 176 patients 
(86 sorafenib, 90 placebo) underwent transarterial chemoembolization (TACE). Patients with prior liver-directed therapy treated with sorafenib had a higher OS (11.9 vs 8.8 months) and longer TTP (5.5 vs 2.8 months) than placebotreated patients, similar to patients with prior TACE therapy who had an OS and TTP of 11.9 and 5.8 months respectively in sorafenib-treated patients compared to 9.9 and 4 months in patients treated with placebo. ${ }^{17}$

\section{Asia-Pacific study}

Another double-blind, placebo-controlled phase III trial was conducted to evaluate the efficacy of sorafenib in HCC patients in the Asia-Pacific region. ${ }^{18}$ Two hundred and seventy one patients from China, South Korea, and Taiwan with CPA liver function class, were randomly assigned to receive either oral sorafenib (400 $\mathrm{mg}$ ) or placebo twice daily. There was no predefined primary endpoint. Overall survival, TTP, TTSP, DCR, and safety were assessed by intention to treat analysis. Median OS was 6.5 months in patients treated with sorafenib, compared to 4.2 months in those who received placebo. Median TTP was 2.8 and 1.4 months in the sorafenib and the placebo group, respectively. Of note the median age of patients in this study was lower than in the SHARP study and a the population of patients with hepatitis B virus (HBV) infection was larger.

Despite a shorter median survival in patients on placebo indicating a worse population than the SHARP study, a prespecified subgroup analysis showed a clinical benefit in all subgroups including patients with worse ECOG PS, vascular invasion, age $\geq 65$ years, macroscopic vascular invasion or extrahepatic spread (or both), and HBV.

\section{Sorafenib in combination with other therapy}

Based on promising results from a phase I trial in patients with advanced hepatocellular carcinoma, ${ }^{19}$ a combination of sorafenib and doxorubicin was evaluated in a randomized phase II trial. Ninety-six patients with advanced HCC received doxorubicin $60 \mathrm{mg} / \mathrm{m}^{2}$ intravenously every 21 days for a maximum of 6 cycles plus either sorafenib $400 \mathrm{mg}$ orally twice daily or placebo. ${ }^{20}$ Patients could continue on single-agent sorafenib or placebo until disease progression. Results of the study showed a longer TTP (8.6 vs 4.8 months) and OS (13.7 vs 6.5 months) in favor of the combination arm. Response rate was modest in both arms of the study (4 vs $2 \%$ ). Grade 3-4 toxicities were higher in the combination arm. A planned intergroup phase II/III study to test the doxorubicin and sorafenib combination versus sorafenib alone is currently underway.

\section{Safety of sorafenib in patients with advanced hepatocellular carcinoma}

$\mathrm{HCC}$ frequently occurs in the setting of chronic liver disease and cirrhosis. For this reason a distinction must be made, whenever possible, between manifestations of worsening chronic liver disease versus drug-related toxicity. Earlier clinical trials with sorafenib reported increased bilirubin levels in some patients receiving the drug, without other evidence to suggest drug-induced liver toxicity.

In the phase I study of sorafenib in patients with advanced refractory nonhematological cancers, ${ }^{8}$ mild diarrhea was the most common treatment-related side effect. The maximum tolerated dose (MTD) was $400 \mathrm{mg}$ twice daily given continuously. Grade 3 or higher dose-limiting toxicities were more common in higher doses and comprised diarrhea ( 2 of 6 patients at $800 \mathrm{mg}$ twice daily), fatigue ( 1 of 6 at $800 \mathrm{mg}$ twice daily), and skin toxicity (4 of 14 at $600 \mathrm{mg}$ twice daily). In the phase I study of sorafenib in 27 Japanese patients with HCC, ${ }^{9} 26$ out of 27 patients (96.3\%) experienced an adverse event, the most common being elevated lipase or amylase which was transient in the majority $(89 \%)$ of patients. Dermatological events were common ( $81.5 \%$ of patients) as were gastrointestinal side effects $(70.4 \%)$. Dermatological events included rash or desquamation in $55 \%$ of patients, and hand-foot syndrome in $44 \%$. Grade 3 or worse toxicities were observed in 23 patients $(85.2 \%)$, most of which were related to laboratory abnormalities. There was no significant difference in the incidence and grade of drug-related adverse events between the CPA and CPB groups in general, but at 400 -mg dose level, diarrhea (62.5 vs $33.3 \%$ ), anorexia ( 50.0 vs $16.7 \%$ ), hypertension ( 37.5 vs $16.7 \%$ ), dry skin ( 37.5 vs $0 \%$ ), and fatigue (25.0 vs $0 \%$ ) were more common in the CPB patients.

In the phase II study of sorafenib in patients with advanced $\mathrm{HCC},{ }^{10}$ the most common drug-related grade 3 adverse events were hand-foot syndrome in 5\%, fatigue in $9.5 \%$, and diarrhea in $8 \%$ of patients. The incidence rates for all adverse events were similar in CPA (97\%) and CPB $(97 \%)$ patients in general. There was no significant difference in the incidence and grade of drug-related adverse events between the CPA and CPB groups in general including fatigue (CPA 41\% vs CPB 37\%), diarrhea (59 \% vs 47\%), and hand-foot syndrome (30\% vs 13\%). CPB patients had 
a higher rate of encephalopathy (CPA 2\% vs CPB 11\%), increased bilirubin (18\% vs 40\%), and worsening ascites (CPA 11\% vs CPB 18\%) when treated with sorafenib. ${ }^{11}$ In the HBV-endemic Asian population specific phase II trial, ${ }^{12}$ diarrhea was the most common toxicity, occurring in $67 \%$ of patients, followed by fatigue (55\%) and hand-foot syndrome (54\%). The majority of patients experienced a transient elevation in AST, ALT, and bilirubin which gradually improved after treatment was discontinued.

The most common adverse events reported in the phase III SHARP trial for sorafenib ${ }^{13}$ were predominantly grade 1 or 2 in severity (Table 2). The overall incidence of serious adverse events from any cause was similar in both groups (52\% vs $54 \%$ in the treatment and the placebo group respectively). Diarrhea, weight loss, hand-foot syndrome, alopecia, anorexia, and voice changes occurred at a significantly higher frequency in the sorafenib group. Grade 3 drug-related adverse events were more common in the sorafenib-treated group and included diarrhea ( $8 \%$ vs $2 \%$ ), hand-foot syndrome ( $8 \%$ vs $<1 \%$ ), hypertension $(2 \%$ vs $<1 \%)$, and abdominal pain ( $2 \%$ vs $1 \%$ ) in sorafenib and placebo group respectively. Grade 3 or 4 laboratory abnormalities were similar in both groups except grade 3 hypophosphatemia (11\% vs $2 \%$ ) and grade 3 or 4 thrombocytopenia ( $4 \%$ vs $<1 \%$ ) which were more common in the sorafenib group.

Drug discontinuation secondary to toxicity was similar in the two study groups (38\% vs 37\%). The most frequent events leading to discontinuation of sorafenib were gastrointestinal events $(6 \%)$, fatigue $(5 \%)$, and liver dysfunction $(5 \%)$. Dose reductions and dose interruptions occurred in $26 \%$ and $44 \%$ of the sorafenib-treated patients versus $7 \%$ and $30 \%$ of patients in the placebo group respectively. Dose reduction in the sorafenib group was secondary to diarrhea, hand-foot syndrome, and rash or desquamation. Treatment was permanently discontinued in $11 \%$ of sorafenib-treated patients versus $5 \%$ of patients treated with placebo group secondary to toxicity.

The incidences of serious hepatobiliary adverse events, serious hemorrhagic events, variceal bleeding, and renal failure $(<1 \%$ and $3 \%)$ were similar in both group. Forty-two deaths not attributed to disease progression were reported within 30 days after the final dose of treatment (13 in the sorafenib group vs 29 in the placebo group).

An exploratory subgroup analysis evaluated sorafenibrelated adverse events stratified by $\mathrm{HCV}$ status, ECOG PS, MVI/EHS, and prior curative treatment. In patients with $\mathrm{HCV},{ }^{14}$ safety profile was similar to the overall study population with manageable grade $1-2$ toxicity. The most common grade 3-4 adverse event in the sorafenib versus placebo arm in HCV-positive patients were hand-foot syndrome (12.9 vs $0 \%$ ), diarrhea (10.8 vs $2.4 \%$ ), hyperbilirubinemia (9.7 vs $2.4 \%$ ), ascites (6.5 vs $9.4 \%$ ), and fatigue (6.5 vs $8.2 \%$ ). Adverse events led to dose reduction in $32 \%$ of sorafenib-treated patients versus $8 \%$ in the placebo group.

The adverse event profile of sorafenib was also similar in patients with PS of 0 compared to PS 1-2. Grade 3-4 drug-related events were diarrhea ( 8.8 vs $8.0 \%$ ), fatigue ( 2.5 vs $5.1 \%$ ), hand-foot syndrome ( 8.8 vs $6.6 \%$ ), hypertension (1.9 vs $1.5 \%$ ), and pain (3.1 vs $2.9 \%$ ) for patients with PS 0 vs PS 1-2 respectively. ${ }^{15}$ Patients with or without MVI/ EHS treated with sorafenib had similar toxicity profile as well

Table 2 Incidence of drug-related adverse events in phase III trials of sorafenib in patients with advanced HCC

\begin{tabular}{|c|c|c|c|c|c|c|c|c|}
\hline \multirow[t]{3}{*}{ Toxicities } & \multicolumn{4}{|l|}{ SHARP } & \multicolumn{4}{|l|}{ Asia-Pacific } \\
\hline & \multicolumn{2}{|l|}{ Sorafenib } & \multicolumn{2}{|l|}{ Placebo } & \multicolumn{2}{|l|}{ Sorafenib } & \multicolumn{2}{|l|}{ Placebo } \\
\hline & Any grade & Grade 3-4 & Any grade & Grade 3-4 & Any grade & Grade 3-4 & Any grade & Grade 3-4 \\
\hline HFS & 21 & 8 & 3 & $<1$ & 45 & 10 & 2.7 & 0 \\
\hline Alopecia & 14 & 0 & 2 & 0 & 25 & 0 & I & 0 \\
\hline Rash/desquam & 16 & 1 & 11 & 0 & 20 & 0.7 & 6.7 & 0 \\
\hline Fatigue & 22 & 4 & 16 & 4 & 20 & 3.4 & 8 & 1.3 \\
\hline Hypertension & 5 & 2 & 2 & I & 19 & 2 & 1 & 0 \\
\hline Weight loss & 14 & $<1$ & 3 & I & 13 & 0 & 2.7 & 0 \\
\hline$N / V$ & 16 & 2 & 11 & 2 & 11 & 0.7 & 10.7 & 1.3 \\
\hline Diarrhea & 38 & 8 & 11 & 2 & 25 & 6 & 5 & 0 \\
\hline Liver dysfunc & $<1$ & $<1$ & 0 & 0 & $<1$ & 0 & 2.7 & 0 \\
\hline Bleeding & 7 & I & 4 & 2 & 2.7 & 0 & 4 & 0 \\
\hline
\end{tabular}

Abbreviations: N/V, nausea/vomiting; HFS, hand-foot syndrome. 
and included diarrhea (5.3 vs $15.7 \%$ ), hand-foot syndrome (6.7 vs $10.1 \%)$, and fatigue (4.8 vs $1.1 \%) .{ }^{16}$

In the phase III trial of sorafenib in Asia-Pacific patients with $\mathrm{HCC},{ }^{18}$ the most frequently reported adverse events (Table 2) were hand-foot syndrome (45\% vs $2.7 \%$ ), diarrhea (25\% vs $5 \%$ ), alopecia ( $25 \%$ vs $1.3 \%)$, fatigue (20\% vs $8 \%$ ), rash or desquamation (20\% vs $6.7 \%)$, hypertension (19\% vs $1.3 \%$ ), and anorexia (13\% vs $2.7 \%$ ). Treatment discontinuation due to adverse events was similar in both groups (19.5\% in treatment group and $13.3 \%$ in the placebo group). Dose reductions were needed in 30.9\% of sorafenib-treated compared to $2.7 \%$ of those treated with placebo. Deaths secondary to serious adverse events were reported in 70 patients (46 in the sorafenib group and 24 in the placebo group). No deaths were considered drug-related.

In the phase II double-blind study of sorafenib plus doxorubicin versus placebo plus doxorubicin in patients with advanced hepatocellular carcinoma, ${ }^{19}$ one patient in the sorafenib group experienced grade 3-4 left ventricular dysfunction. Fatigue and neutropenia were the most commonly reported grade 3-4 adverse effects, and they occurred equally in both groups of patients.

\section{Conclusion}

Sorafenib is a novel drug that targets growth factor receptors known to play a role in the pathophysiology of HCC. Sorafenib demonstrated antitumor activity as first-line therapy in patients with advanced HCC. It demonstrated a modest survival benefit over placebo in 2 phase III trials, especially in patients with CPA liver disease. Sorafenib has an acceptable toxicity profile that includes hand-foot syndrome, rash, fatigue, hypertension, and diarrhea.

Given the complexity of the interactions between the pathways involved in tumoregensis, targeted therapies with different mechanisms of action are being combined in the treatment of HCC in order to inhibit numerous pathways. Several clinical trials are currently evaluating sorafenib in combination with targeted agents in the treatment of HCC; these agents include insulin growth factor receptor inhibitor, bevacizumab, erlotinib, and angiopoietin 1-2-neutralizing peptibody among others. Studies evaluating the combination of sorafenib and chemotherapeutic agents are also underway. Prognostic and predictive markers need to be developed to identify patients with advanced HCC who may or may not respond to sorafenib.

Patients with localized disease who have undergone resection or liver transplantation are prone to relapse and need effective adjuvant therapy. Currently no adjuvant therapy is recommended for high-risk patients. Clinical trials evaluating the role of sorafenib after, or concurrently with, TACE, selective internal radiation (SIR)-spheres, and radiation are also underway.

\section{Disclosures}

The authors declare no conflicts of interest.

\section{References}

1. Parkin DM, Bray F, Ferlay J, Pisani P. Global cancer statistics, 2002. CA Cancer J Clin. 2005;55:74-108.

2. Llovet JM, Burroughs A, Bruix J. Hepatocellular carcinoma. Lancet. 2003;362(9399):1907-1917.

3. Wilhelm S, Carter C, Lynch M, et al. Discovery and development of sorafenib: a multikinase inhibitor for treating cancer. Nat Rev Drug Discov. 2006;5(10):835-844.

4. Ito Y, Sasaki Y, Horimoto M, et al. Activation of mitogen-activated protein kinases/extracellular signal-regulated kinases in human hepatocellular carcinoma. Hepatology. 1998;27:951-958.

5. Villanueva A, Newell P, Chiang DY, Friedman SL, Llovet JM. Genomics and signaling pathways in hepatocellular carcinoma. Semin Liver Dis. 2007;27:55-76.

6. Calvisi DF, Ladu S, Gorden A, et al. Ubiquitous activation of Ras and Jak/Stat pathways in human HCC. Gastroenterology. 2006;130: $1117-1128$.

7. Semela D, Dufour JF. Angiogenesis and hepatocellular carcinoma. J Hepatol. 2004;41:864-880.

8. Strumberg D, Voliotis D, Moeller JG, et al. Results of phase I pharmacokinetic and pharmacodynamic studies of the Raf kinase inhibitor BAY 43-9006 in patients with solid tumors. Int J Clin Pharmacol Ther. 2002;40(12):580-581.

9. Furuse J, Ishii H, Nakachi K, Suzuki E, Shimizu S, Nakajima K. Phase I study of sorafenib in Japanese patients with hepatocellular carcinoma. Cancer Sci. 2008;99(1):159-165.

10. Abou-Alfa GK, Schwartz L, Ricci S, et al. Phase II study of sorafenib in patients with advanced hepatocellular carcinoma. J Clin Oncol. 2006;24(26):4293-4300.

11. Abou-Alfa GK, Amadori D, Santoro A, et al. Is sorafenib (S) safe and effective in patients (pts) with hepatocellular carcinoma (HCC) and Child-Pugh B (CPB) cirrhosis? Clin Oncol. 2008;26:(May 20 Suppl): abstr 4518.

12. Yau T, Chan P, Ng KK, et al. Phase 2 open-label study of single-agent sorafenib in treating advanced hepatocellular carcinoma in a hepatitis B-endemic Asian population: presence of lung metastasis predicts poor response. Cancer. 2009;115(2):428-436.

13. Llovet J, Ricci S, Mazzaferro V, et al. Sorafenib in advanced hepatocellular carcinoma. N Engl J Med. 2008;359(4):378-390.

14. Bolondi L, Caspary W, Bennouna J, et al. Clinical benefit of sorafenib in hepatitis C patients with hepatocellular carcinoma (HCC): Subgroup analysis of the SHARP trial. Gastrointestinal Cancers Symposium, Orlando, Florida; 2008:abstr 129.

15. Raoul J, Santoro A, Beaugrand M, et al. Efficacy and safety of sorafenib in patients with advanced hepatocellular carcinoma according to ECOG performance status: A subanalysis from the SHARP trial. Clin Oncol. 2008;26(May 20 Suppl):abstr 4587.

16. Sherman M, Mazzaferro V, Amadori D, et al. Efficacy and safety of sorafenib in patients with advanced hepatocellular carcinoma and vascular invasion or extrahepatic spread: A subanalysis from the SHARP trial. J Clin Oncol. 2008;26:(May 20 Suppl):abstr 4584.

17. Galle PR, Blanc J, Van Laethem JL, et al. Efficacy and safety of sorafenib in patients with advanced hepatocellular carcinoma and prior anti-tumor therapy: a sub-analysis from the SHARP trial. 43rd annual meeting of the European Association for the Study of the Liver (EASL 2008). Milan, Italy; 2008 April 23-27. 
18. Cheng AL, Kang YK, Chen Z, et al. Efficacy and safety of sorafenib in patients in the Asia-Pacific region with advanced hepatocellular carcinoma: a phase III randomised, double-blind, placebo-controlled trial. Lancet Oncol. 2009;10(1):25-34.

19. Richly H, Schultheis B, Adamietz IA, et al. Combination of sorafenib and doxorubicin in patients with advanced hepatocellular carcinoma: results from a phase I extension trial. Eur J Cancer. 2009;45(4): 579-587.
20. Abou-Alfa GK, Johnson P, Knox J, et al. Final results from a phase II (PhII), randomized, double-blind study of sorafenib plus doxorubicin $(\mathrm{S}+\mathrm{D})$ versus placebo plus doxorubicin $(\mathrm{P}+\mathrm{D})$ in patients $(\mathrm{pts})$ with advanced hepatocellular carcinoma (AHCC). Gastrointestinal Cancers Symposium, Orlando, Florida; 2008:abstr 128.

\section{Publish your work in this journal}

OncoTargets and Therapy is an international, peer-reviewed, open access journal focusing on the pathological basis of all cancers, potential targets for therapy and treatment protocols employed to improve the management of cancer patients. The journal also focuses on the impact of management programs and new therapeutic agents and protocols on

\section{Dovepress}

patient perspectives such as quality of life, adherence and satisfaction. The manuscript management system is completely online and includes a very quick and fair peer-review system, which is all easy to use. Visit http://www.dovepress.com/testimonials.php to read real quotes from published authors.

Submit your manuscript here: http://www.dovepress.com/oncotargets-and-therapy-journal 